\title{
Strategic Efforts to Diagnose And Treat Osteochondroma
}

\author{
Dr. Parveen Chandna*, Dr.Pramod Setty J.**, Dr. Jeevika M.U.***, Dr. \\ Siddesh M.B.**** Dr. Kishore Chautakuri*, \\ * Pg In Radio-Diagnosis, **Ex. Hod, *** Professor \& Hod, ****Asso.Professor Dept. Of Radio-Diagnosis, \\ J.J.M. Medical College, Davangere.
}

\begin{abstract}
Osteochondroma or osteocartilagenous exostosis is not only a common tumour of oral and maxillofacial skeleton but certainly regarded as the most common benign tumour of bone. Osteochondroma constitutes approximately 10-15\% of all bone tumours and 20-50\% of all benign tumours. Osteochondroma is an exophytic lesion that originates from the cortex of the bone and is cartilage covered lesion.

The pathogenesis of osteochondroma is still controversial as the same has also been considered to be a part of an inherited syndrome. Porter and Simpson suggested that a genetic component might also be involved in the neoplastic pathogenesis. The most commonly accepted view is a metaplastic change of periosteum and the osteochondral layer in condyle leading to production of cartilage which subsequently ossifies.

Osteochondroma of the mandibular condyle is relatively rare,slow growing benign tumour and may reveal signs and symptoms similar to those observed in patients with temporomandibular joint (TMJ) dysfunction. It is a well recognized entity in the appendicular skeleton especially long bones, such as the proximal metaphysis of the tibia and distal metaphysis of the femur, but it is an uncommon benign tumour of jaws. The imaging pathognomic characteristics of this tumour is the cortical and medullary continuity of the lesions with the adjacent bone.Most gnathic examples are observed in the condyle, coronoid process and very rarely in tongue. Reported prevalence of osteochondromas in craniofacial region is $0.6 \%$.
\end{abstract}

\section{Introduction}

Osteochondroma represents the most common pseudotumoral bone lesion with current concepts of bone tumours consider osteochondroma as a development or hamartomatous lesions rather than a true neoplasm This lesion may be existing in a solitary fashion or as multiple osteochondromas as an integral part of an inherited syndrome that results in disturbances of skeletal growth and development. These lesions may also appear with complications of bone deformities fractures ,neurological or vascular compromise. The following report gives details of an unusual case of osteochondroma.

Ultrasonography has been used to assess the cartilage cap and associated complications such as arterial or venous thrombosis, aneurysm and bursitis but had not been used till now to assess the underlying bone. Angiography is a gold standard for assessing the vascular occlusion and malignant nature of lesion but is still invasive.

It is imperative to perform regular clinical and radiological examinations after surgery to detect any signs of recurrence following reporting of chief complaints of progressive asymmetry of face, difficulty in mastication and disturbance of mouth opening. Preoperative CT scans and axial CT scan revealed emerging bony masses on the both sides of condylar surface.Tumour and inflammation have also been suggested as contributory factors. Osteochondroma is a slow growing benign tumour of the axial skeleton mostly arises from metaphyseal regions of long bones.Most commonly seen in $2^{\text {nd }}$ and $3^{\text {rd }}$ decade of life Common clinical manifestations of the osteochondroma of the mandibular condyle include facial asymmetry, pain and disturbance of mouth opening, difficulty in mastication and malocclusion.

\section{Casereport :}

A 12 year female patient was referred to Bapuji Dental OPD for diagnosis and treatment of facial asymmetry ,due to deviation of mandible to left side with major complaints of pain and disturbance in opening the mouth ,difficulty in mastication and painfully impaired mobility of the right temporomandibular joint.On clinical examination visible swelling in the right preauricular region was also observed.The patient was conscious of swelling due to its slow increase in size .

\section{Ct findingsreveals :}

A partially exophytic mass measuring $12 \times 9 \mathrm{~mm}$ arising from the condylar process of the right mandible. The exophytic lesion seems to have corticomedullary continuation with the parent bone. Temporomandibular joints asymmetry noted. No evidence of scattered or irregular calcifications noted within the bony lesion. There are no features of bony erosions, adjacent soft tissue mass or destruction of the adjacent bone as 
such. Rest of the visualized bones appear to be normal. Features suggestive of Sessile Osteochondroma involving condylar process of the right mandible

An osseous tumour of the condylar process of right mandible was depicted on conventional radiographs

MRI findings no abnormal marrow signal intensity seen.

Old known case of Sessile Osteochondroma involving condylar process of the right mandible has also been a moot point of discussion.

\section{Conclusion}

A careful assessment of the patients history as detailed and knowledge pertaining to findings of this lesion might provide valuable information for the diagnosis and treatment of facial asymmetry. Imaging techniques is more valuable for accurately diagnosing and determining treatment in detection of these lesions as it presents some typical radiological features, mainly cortical and medullary continuity. The presence of desmal ossified bones in facial skeleton is oneof the reason that osteochondromas are rarely found in facial skeleton.The regulation of osseous growth in condylar hyperplasia has been related to insulin like growth factor -1 receptor expression.Histologically, the diagnosis of an osteochondroma includes chondrocytes of the cartilaginous cap arranged in clusters parallel to lacunar space.Computed tomography with three-dimensional (3D) reconstruction showed rounded bony mass projects out from the condylar process of the right mandible which clearly depicts size, morphology, margins and directions of the growth of lesion that helps in surgical management.
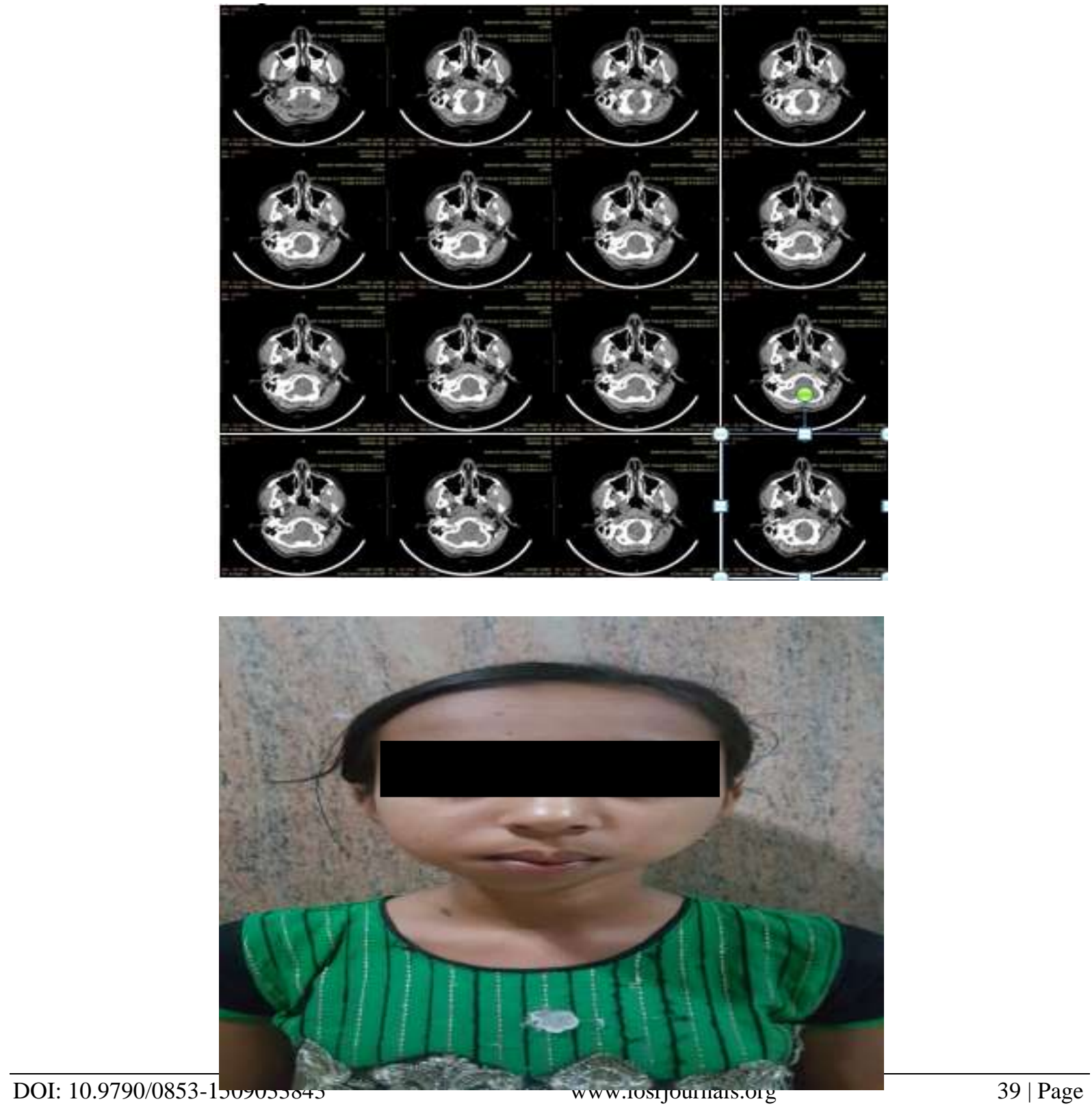

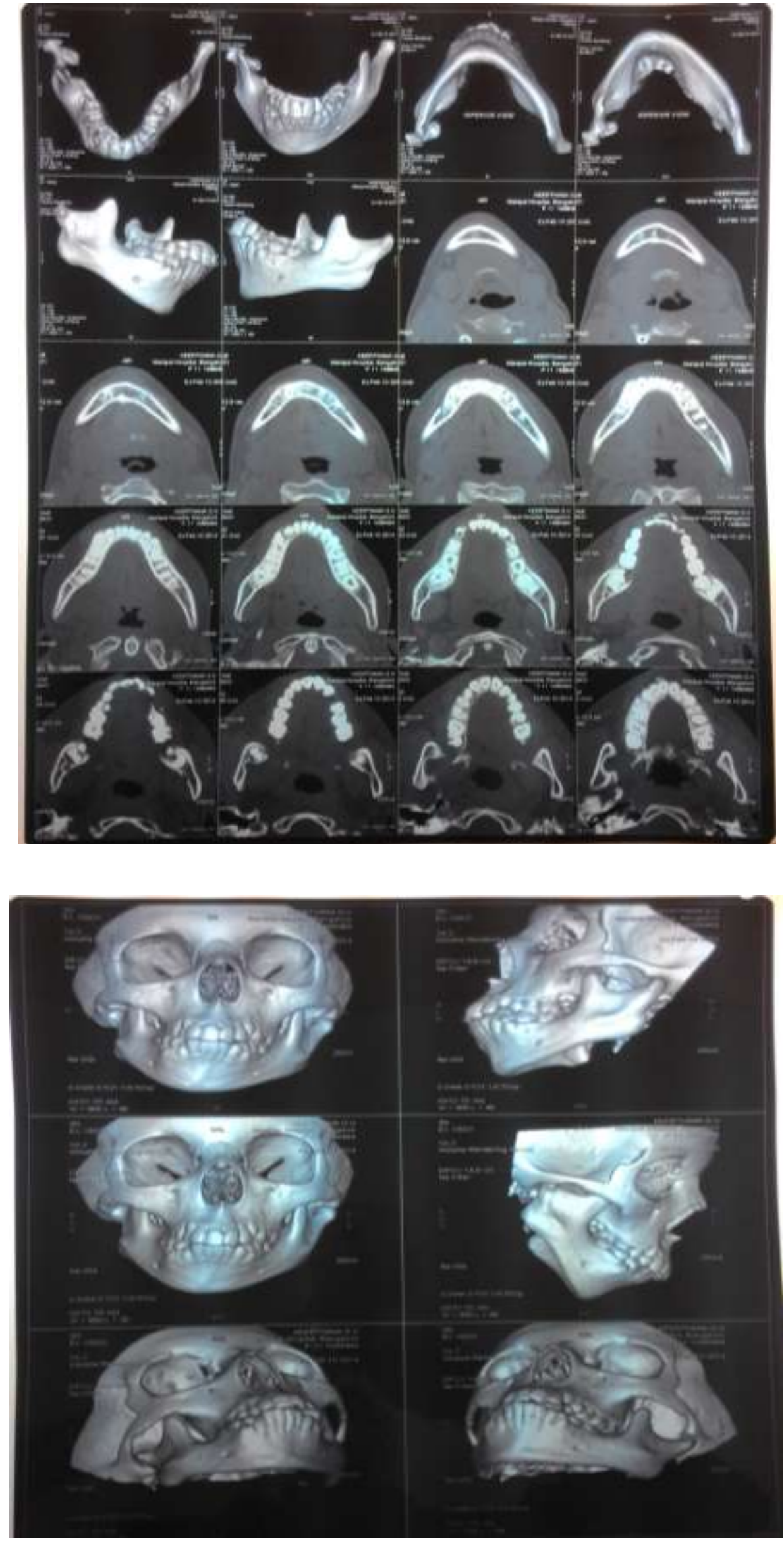


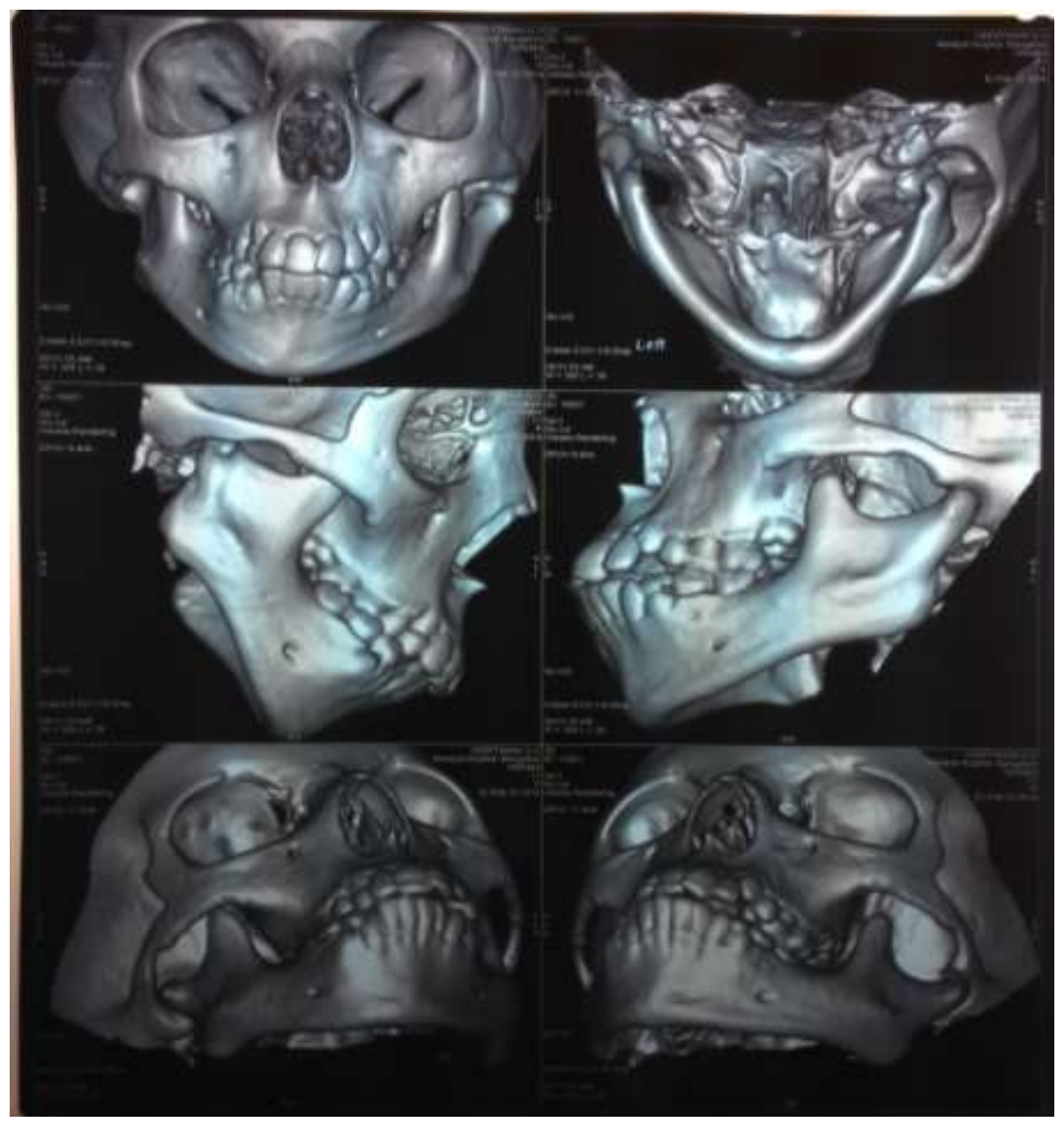




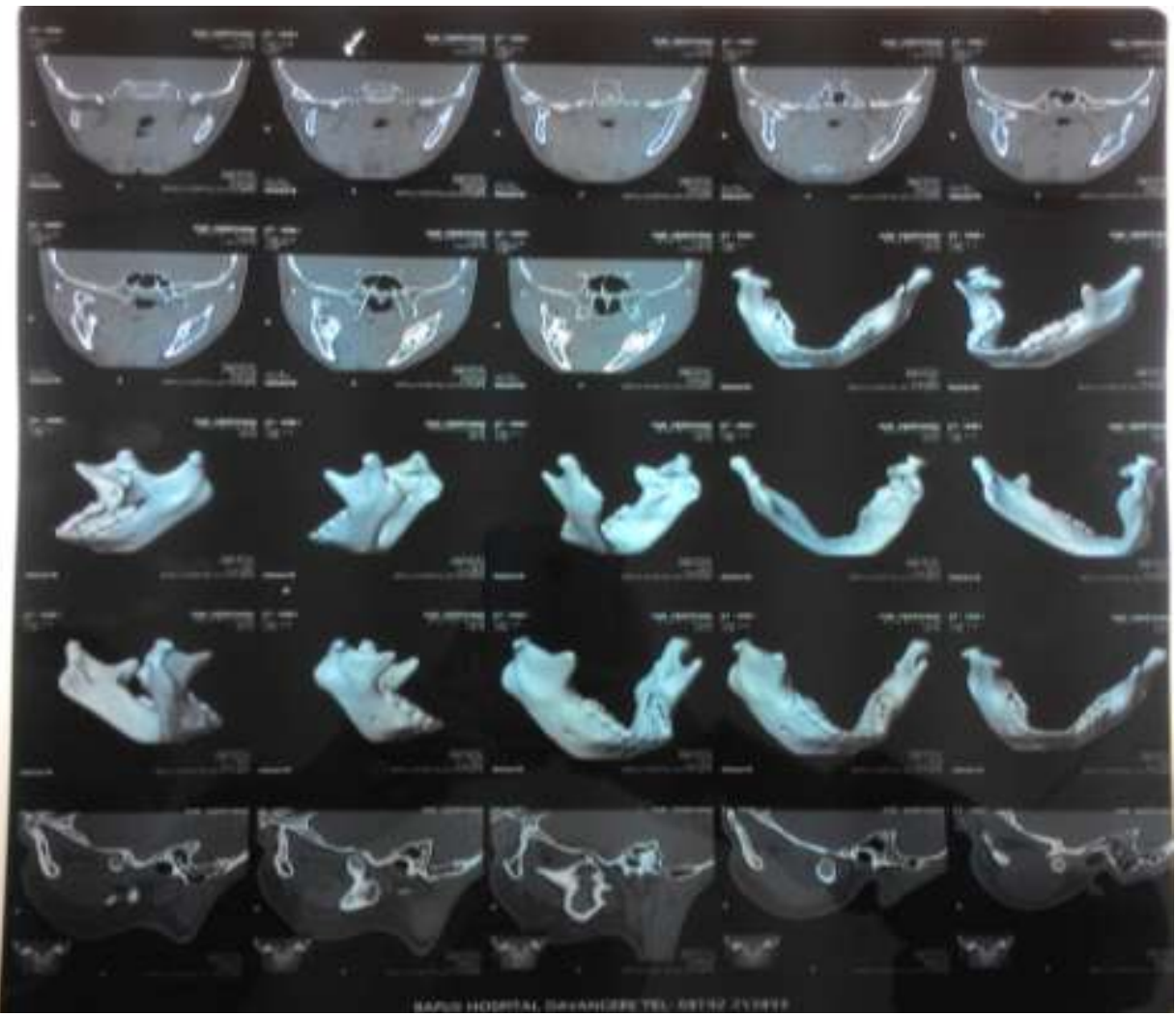




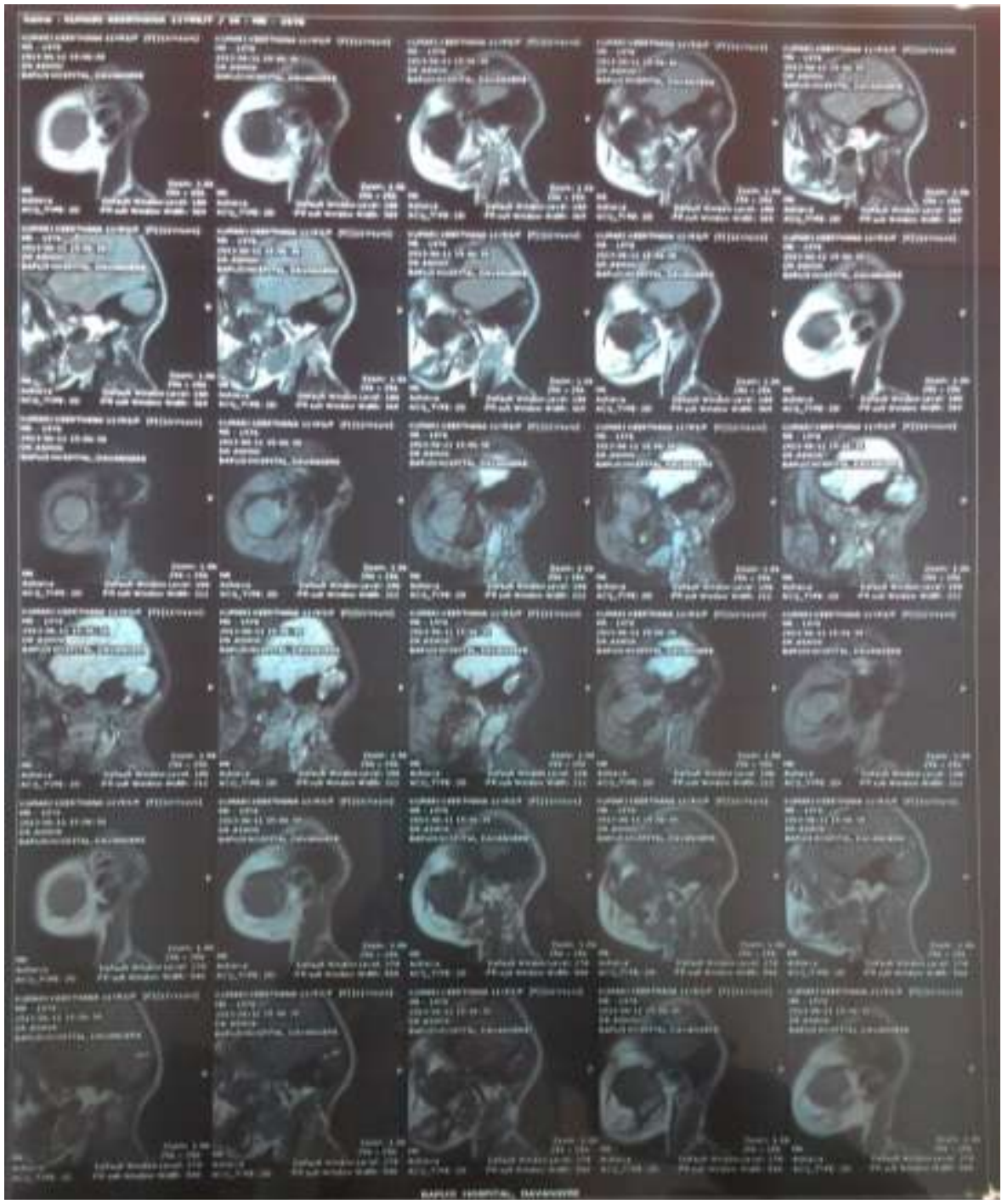




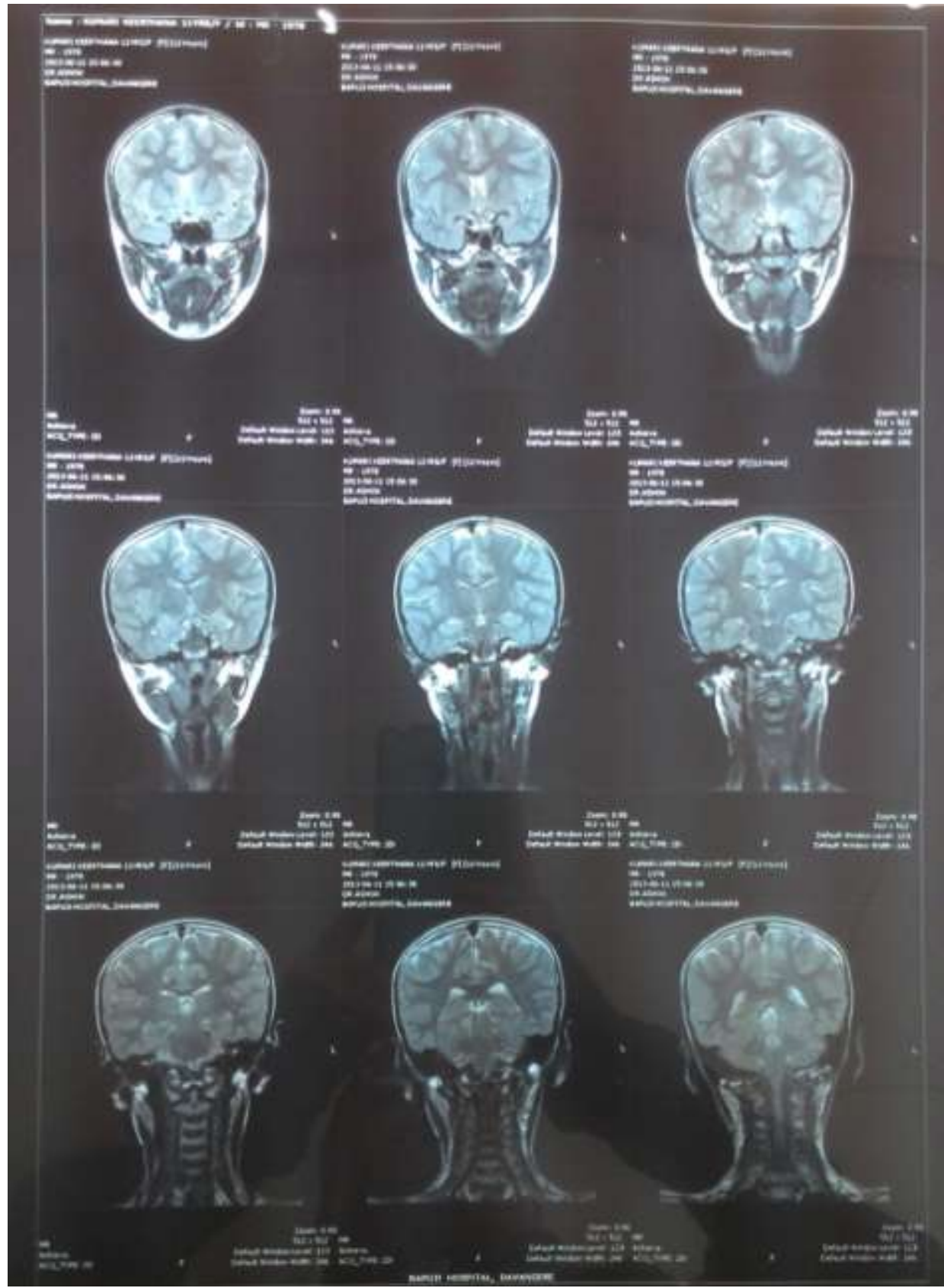

